\title{
El carvedilol reduciría más la mortalidad que el metoprolol en la insuficiencia cardíaca
}

Comparison of carvedilol and metoprolol on clinical outcomes in patients with chronic heart failure in the Carvedilol Or Metoprolol European Trial (COMET):randomised controlled trial. Poole-Wilson PA, Swedberg K, Cleland JFK, et al, for the COMET investigators Lancet 2003; 362:7-13.

\section{Objetivo}

Comparar los efectos del carvedilol y el metoprolol sobre la mortalidad y morbilidad en pacientes con disminución de la fracción de eyección y síntomas de insuficiencia cardíaca.

Diseño

Ensayo clínico controlado multicéntrico, aleatorizado y doble ciego. Lugar

Participaron 341 centros pertenecientes a 15 países europeos.

Pacientes

Se incluyeron 3029 pacientes con insuficiencia cardíaca (IC) clase funcional (CF) II-IV estable y al menos un internación de causa cardiovascular en los dos años previos, óptimamente tratados (salvo intolerancia) con diuréticos e inhibidores de la enzima convertidora de angiotensina (IECA) y una fracción de eyección (FE) $<35 \%$.

\section{Intervención}

Los pacientes fueron aleatoriamente asignados a recibir carvedilol $(n=1511)$ o tartrato de metoprolol $(n=1518)$. Las dosis se aumentaban cada dos semanas (según la tolerancia) con carvedilol: $3.125,6.25,12.5$ y $25 \mathrm{mg}$ (dosis buscada) dos veces al día y con metoprolol: $5,12.5,25$ y $50 \mathrm{mg}$ dos veces al día.

\section{Medición de resultados principales}

Se consideraron dos resultados principales: mortalidad total y resultado combinado de mortalidad total más internación por cualquier causa. El análisis fue por intención de tratar.

\section{Resultados principales}

El seguimiento promedio fue de 4,8 años, y completo en el $99 \%$ de los pacientes.El promedio de edad fue 62 años, y de FE $26 \%$. La dosis promedio utilizada de carvedilol fue $41,8 \mathrm{mg}$ /día y de metoprolol $85 \mathrm{mg} /$ día.La frecuencia cardíaca con carvedilol fue -1,6 (IC95\% $-3,2 \mathrm{a}-0,4, \mathrm{p}<0.001$ ) latidos/minuto y la tensión arterial sistólica $-1,8$ (IC95\% -3,2 a -0,4, p<0.001) respecto al metoprolol.El carvedilol redujo significativamente la mortalidad total en un $27 \%$ comparado con metoprolol (ver $\mathrm{HR}^{*}$ en tabla) haciéndose ostensible el beneficio a los 6 meses. La dirección y magnitud de este resultado se mantuvo en todos los subgrupos prefijados (sexo, edad, CF, causa, FE, frecuencia cardíaca, tensión arterial y diabetes).

\begin{tabular}{l|c|c|c|c|c} 
Resultado & $\begin{array}{c}\text { Carvedilol } \\
\mathbf{n = 1 5 1 1}(\%)\end{array}$ & $\begin{array}{c}\text { Metoprolol } \\
\mathbf{n = 1 5 1 8}(\%)\end{array}$ & $\begin{array}{c}\text { RRA } \\
(\%)\end{array}$ & $\begin{array}{c}\text { NNT (IC 95\%) } \\
\text { a 5 años\# }\end{array}$ & $\begin{array}{c}\text { HR }^{*} \\
(\mathbf{I C ~ 9 5 \% )}\end{array}$ \\
\hline Mortalidad Total & $512(34)$ & $600(40)$ & 5,7 & $18(11$ a 45) & $0,83(0,74-0,93)$ \\
\hline Mortalidad CV & $438(29)$ & $534(35)$ & 6,2 & $16(11$ a35) & $0,80(0,70-0,90)$ \\
\hline $\begin{array}{l}\text { Muerte 0 } \\
\text { Internación }\end{array}$ & $1116(74)$ & $1160(76)$ & 2,6 & & $0,94(0,86-1,02)$ \\
\hline
\end{tabular}

RRA Reducción de riesgo absoluto, RR Riesgo Relativo, NNT Número Necesario a Tratar, HR Hazard Ratio \# Calculados por EVIDENCIA

Conclusiones

El carvedilol mejora la sobrevida en relación al metoprolol en pacientes con IC tratados con IECA y diuréticos.

Fuente de financiamiento: F Hoffmann La Roche and GlaxoSmithKline.

\section{Comentario}

El presente estudio permite estimar que el carvedilol mejora la sobrevida respecto al metoprolol en 1,4 años (IC95\% 0,5-2,3) en pacientes con IC y disfunción sistólica óptimamente tratados. EI NNT de 59 pacientes-año para prevenir una muerte es similar al de IECA o bloqueantes $\beta$-1 selectivos (metoprolol, bisoprolol) frente al placebo.Esta reducción fue tanto para mortalidad total como para mortalidad cardiovascular, muerte súbita (14 vs $17 \%$ ) y muerte por falla circulatoria (8 vs $13 \%$ ).

El estudio incluyó pacientes estables, y el $97,5 \%$ de los mismos se encontraba en clase funcional II-III.Es por ello que no pueden extraerse conclusiones en relación a pacientes con insuficiencia cardíaca severa. La mortalidad anual de este estudio fue $8,3 \%$ con carvedilo y $10 \%$ con metoprolol, la cual está por encima del $7,2 \%$ alcanzado con metoprolol en el MERIT-HF y del 8,8\% con bisoprolol en el CIBISII2 y por debajo del $11,4 \%$ del COPERNICUS ${ }^{3}$ con carvedilol (todos con similar beneficio del $35 \%$ vs placebo). Aunque la población del COMET sea más grave, estuvo equilibradamente distribuida entre grupos por lo que no podría explicar las diferencias, pero ¿por qué la rama metoprolol del COMET tuvo peor eficacia que en los estudios previos de metoprolol? Cabe entonces preguntarse si las dosis fueron las mismas que en otros estudios.El MERIT-HF prefijó 200 $\mathrm{mg} / \mathrm{día}$ de succinato de metoprolol CR/XL (de acción prolongada) equivalente según los autores a $130 \mathrm{mg} /$ día de tartrato de metoprolol y alcanzando $106 \mathrm{mg}$ de esta equivalencia, el MDC4 alcanzó 108 $\mathrm{mg} / \mathrm{día}$ vs los $85 \mathrm{mg} / \mathrm{día}$ del COMET. En el subgrupo de dosis baja de metoprolol del MERIT-HF ( $51 \mathrm{~m} \mathrm{~g} /$ día) la reducción de mortalidad fue similar al de alta dosis (128 $\mathrm{mg} /$ día). Esta respuesta plana del metoprolol y las escasas diferencias de frecuencia cardíaca entre los grupos del COMET (que desaparecieron a los 16 meses) hace poco probable que la menor dosis alcanzada de metoprolol explique totalmente la superioridad del carvedilol pero sí una parte, dado el menor bloqueo $\beta$-1.Otra explicación radica en las características distintivas del carvedilol: $\alpha$ y $\beta$-bloqueante no selectivo, antioxidante, que incrementa la sensibilidad a la insulina. Esta plausibilidad biológica también cuenta con ciertas evidencias clínicas que empezaron a vislumbrarse en el meta-análisis de Lechat ${ }^{5}$ que mostró que la reducción en mortalidad total era mayor con carvedilol que con los $\beta$-1selectivos $(49 \%$ versus $18 \%, p=0,049)$.El diferente perfil farmacológico de los $\beta$-bloqueantes podría explicar la falta de beneficios en mortalidad del Bucindolol ${ }^{6}$ o el mayor incremento de la FE con carvedilol que con metoprolol.? Cabe destacar que los beneficios del carvedilol se encontraron no frente a placebo, sino frente a otra droga de probada eficacia como el metoprolol.Queda por determinar ante igualdad de bloqueo $\beta$-1 si estos beneficios se mantendrían o extenderían a los pacientes en CF IV o con falla sistólica asintomática;y por explicar la falta de acción sobre las internaciones. Quizás un estudio pragmático* de carvedilol, metoprolol y bisoprolol a las máximas dosis toleradas en una cohorte de pacientes representativos de la práctica satisfaría más a los médicos y sería de gran ayuda para conocer la verdad de los $\beta$-bloqueantes en la IC. ${ }^{8}$

Conclusión del comentador: aun con limitaciones, siendo el úni co estudio comparativo, bien diseñado, que mostró una posible superioridad del carvedilol frente a un $\beta$-1-bloqueante como el metoprolol, es razonable que se transforme en b-bloqueante de elección para la IC mientras no existan estudios contrapuestos;pero un mensaje aun más importante es que todo paciente con IC reciba al menos un $\beta$-bloqueante a la máxima dosis tolerada.

*Ver glosario

\section{Dr. Agustín Ciapponi [ Unidad de Medicina Familiar y Preventiva. Hospital Italiano de Buenos Aires ]}

\section{Referencias}

1.Effect of metoprolol CR/XL in chronic heart failure: metoprolol CR/XL Randomised Intervention Trial in Congestive Heart Failure (MERIT-HF) MERIT-HF Study Group.Lancet 1999;353:2001-7. 2. The cardiac insufficiency bisoprolol study II (CIBIS II).CIBIS II Ivestigators and Committees. Lancet 1999;353:9-13.

3. Packer M, Coats AJ, Fowler MB, et al. Effect of carvedilol on survival in severe chronic heart failure.N Engl J Med 2001; 344:1651-58.

4. Waagstein F, Bristow MR, Swedberg K, et al.Beneficial effects of metoprolol in idiopathic dilated cardiomyopathy.Lancet 1993;342:1441-46.

5. Lechat P, Packer M, Chalon S et al.Clinical effects of beta-adrenergic blockade in chronic heart failure: a meta-analysis of double-blind, placebo-controlled, randomized trials.Circulation 1998;98(12):1184-91. 6. The Beta-Blocker Evaluation of Sunvival Trial Investigators. A trial of the beta-blocker bucindolol in patients with advanced chronic heart failure.N Engl J Med 2001:344:1659-67.

7. Packer M, Antonopoulos GV, Berlin JA, Chittams J, Konstam MA, Udelson JE. Comparative effects of carvedilol and metoprolol on left ventricular ejection fraction in heart failure: results of a meta7. Packer M, Antonopoulos GV, Berlin JA 8. Dargie JH. B-blockers in heart failure.Lancet 2003;362:2 